\title{
Un estudio de caso de la estrategia administrativa basada en comités
}

\author{
Enrique Vílchez, Quesada \\ Profesor de la Escuela de Informática de la Universidad Nacional de Costa Rica \\ Heredia, Costa Rica \\ Gaby Ulate Solís \\ Profesora de la División de Educología de la Universidad Nacional de Costa Rica \\ Heredia, Costa Rica \\ Ricardo Monge Gapper \\ Profesor Universidad Interamericana \\ San José, Costa Rica
}

Recibido 4 de mayo de 2007 • Aprobado 31 de julio de 2008

\begin{abstract}
Resumen. Se aplica la teoría de administración curricular para explicar el uso de la estrategia de gestión por comités en las instituciones de enseñanza general básica y diversificada. Se realiza un estudio de caso en una institución educativa del casco metropolitano, con características organizacionales comunes a una realidad nacional que incide en la mala praxis de esta estrategia. Se establecen dentro del estudio de caso, algunas de las causas de su mal uso en el contexto particular de la institución educativa UPJRAR, ubicada en Tibás y a la luz de estos resultados, se propone una serie de recomendaciones para mejorar la utilización de la estrategia administrativa basada en comités.
\end{abstract}

Palabras clave. Administración, educación, estrategia, comités, instituciones.

Abstract. The theory of curricular administration is applied in order to explain the use of the strategy of management committees in institutions of elementary and high school education. A case study is carried out in an educational institution of the metropolitan area; an institution known for organizational features that are common in a national reality that affects the malpractice of this strategy. It is established within the case study, some of causes of its abuse in the particular context of the educational institution "Unidad Pedagógica José Rafael Araya Rojas" located in the "Tibás, La Florida" community and in the light of these results, some recommendations are suggested in order to improve the use of the administrative strategy based on committees.

Key words. Administration, education, strategy, committees, institutions.

\footnotetext{
Posee experiencia docente en campos diversos, tales como: la matemática y sus aplicaciones, tecnologías de información y comunicación y aprendizaje virtual. Correo electrónico: evilchez@una.ac.cr

2 Posee amplia experiencia docente en el área educativa, en general, y en la enseñanza de las artes y la comunicación visual, específicamente. En la actualidad, colabora con la División de Educología en la actividad de investigación en docencia denominada: "Propuesta del curso Recursos didácticos para el aprendizaje bajo un enfoque bimodal”. Correo electrónico: gabyulate@hotmail.com

3 Su área de formación: Ingeniero en Sistemas de Información, estudiante de Doctorado en Aplicaciones de la Informática de la Universidad de Alicante, España.
} 


\section{INTRODUCCIÓN}

Uno de los hechos innegables en los centros de educación primaria y secundaria en nuestro país, es la delegación de algunas labores administrativas y políticas, en comités formados por los profesores y las profesoras de la institución.

Los comités constituyen una estrategia de administración y como tal, es necesario que responda a una serie de características y requerimientos relacionados, principalmente, con el trabajo de equipo. Los integrantes de un comité deben estar dispuestos a colaborar, y sus dirigentes a propiciar la participación de todos, definir roles y responsabilidades, tomar decisiones objetivas, coordinar, dar confianza e implicación y establecer un plan de actuación, todo con la finalidad de garantizar el cumplimiento de las funciones y los objetivos para los que fueron diseñados.

El problema radica cuando se aprecia en las instituciones educativas la aplicación de esta estrategia en términos reales, pero que, con frecuencia, los comités se limitan a ocupar un espacio de lectura en el plan institucional, sin existir un proceso sistemático de operacionalización de funciones y de logro de objetivos.

¿Por qué ocurre esto en los centros de enseñanza?, ¿cómo podría minimizarse tal situación?, ¿cuáles recomendaciones se podrían seguir para mejorar la utilización de esta estrategia administrativa? Con este trabajo se pretende evaluar la funcionalidad de la estrategia administrativa por comités, la idea principal ha consistido en realizar un estudio de caso, tomando como fuente primaria de información el UPJRAR ubicado en la provincia de San José, cantón de Tibás. Se analiza el uso y el funcionamiento de los comités en esta institución, y se trata de responder, de forma inductiva, a las interrogantes anteriormente planteadas.

\section{REFERENTE TEÓRICO}

\section{La estrategia administrativa basada en comités}

El mejoramiento de la calidad de la educación, constituye una de las preocupaciones más importantes en el quehacer educativo de nuestra política educativa. El proceso de planificación del trabajo que desarrolla una organización educativa, constituye el procedimiento mediante el cual, se relacionan, se ordenan y se diseñan acciones para lograr determinados propósitos, con el fin de darle respuesta a las necesidades e intereses de la comunidad estudiantil: cuerpos docente y administrativo.

Planificar significa: "hacer explícita, de manera adecuada, la intención de nuestras acciones, mediante un proceso de previsión de necesidades y racionalización del empleo de los medios materiales y recursos humanos disponibles, con el propósito de alcanzar objetivos concretos en períodos de tiempo específicos" (Murillo, 1995, p. 27).

La planificación es un proceso, no es sólo un conocimiento teórico, sino, también, una técnica operativa, es decir, una serie de conocimientos, métodos, procedimientos, técnicas de investigación, organización y administración, que tienen un efecto directo sobre las necesidades inmediatas ante una determinada situación.

Particularmente, la planificación educativa se entiende como un proceso que se realiza con base en las siguientes etapas: diagnóstico, programación, ejecución y evaluación.

La etapa de diagnóstico, tiene como finalidad develar las áreas más deficientes, permitiendo determinar las prioridades de programación de acciones tanto preventivas como de mejoramiento. 
La etapa de programación, incluye uno de los aspectos más importantes de cualquier proceso de planeamiento; la formulación de los objetivos que se persiguen en un plazo establecido en el proyecto de planificación (en función de las necesidades educativas diagnosticadas). En esta etapa, también se concretan los recursos materiales y humanos con que se cuenta para el alcance de los objetivos, y se elaboran los planes metódicos de acción en congruencia con las decisiones gubernamentales, para, finalmente, ser ejecutadas por los organismos administrativos responsables.

La última etapa de un ciclo de planificación, incluye la evaluación del proceso. La evaluación consiste en un juicio objetivo y crítico de revisión permanente acerca de las decisiones y los medios de acción involucrados en los proyectos de planificación educativa. Fundamentalmente, abarca tres etapas:

1. El estudio de los efectos: esta etapa se caracteriza por la observación de los impactos concretos que ha ocasionado el proceso de ejecución, en especial, los efectos inesperados.

2. El juicio de los objetivos: se refiere a la comparación entre los resultados obtenidos y los objetivos buscados.

3. La innovación experimental: la idea de esta etapa consiste en generar las soluciones alternativas de las fallas del proceso.

En las circunstancias actuales de un país subdesarrollado como el nuestro, la planificación exige un lugar privilegiado en todos los ámbitos. Por ser muchas las necesidades y escasos los recursos, se hace indispensable organizar acciones inteligentes para que con el menor desperdicio de tiempo y esfuerzo, se logren resultados eficientes y seguros.

Particularmente, en el campo educativo la planificación es el medio más viable para poder mejorar, en alguna medida, los graves deterioros que ha sufrido su calidad como consecuencia de la crisis financiera.

En las instituciones de enseñanza media, los procesos administrativos, sin duda, se fundamentan en la planificación educativa. Tradicionalmente, se ha utilizado para ello una técnica social denominada FODA, la cual pretende hacer un diagnóstico y una formulación de posibles soluciones a problemas localizados. Con el FODA se formulan, con claridad, las metas, la misión y la visión de la institución educativa. La principal característica del FODA, es la posibilidad de hacer planteamientos colectivos, holísticos y consensuados, para la consecución de soluciones a los problemas apremiantes. Posibilita el trabajo en equipo y el logro del compromiso por parte de los involucrados, por medio de la potenciación de los recursos personales, humanos y materiales de una mejor óptima. Al ser una estrategia de planificación educativa cumple o debería cumplir con las etapas de: diagnóstico, programación, ejecución y evaluación.

La etapa de diagnóstico del FODA se lleva a cabo mediante una lluvia de ideas sobre las debilidades, las fortalezas, las amenazas y las oportunidades que se observan y se sienten. De esta etapa, es necesario pasar a una identificación de prioridades, según las debilidades detectadas y la magnitud de su importancia. Posteriormente, al tener claro el panorama de recursos y proyectos para la solución de los problemas priorizados, se procede a la etapa de programación, dividiendo las necesidades diagnosticadas en grupos de trabajo denominados comités.

Los comités se encargan de definir los objetivos, las actividades y los mecanismos de evaluación de cumplimiento. Además, organizan cada actividad mediante una calendarización en la que se eligen los responsables y la forma como se lograrán alcanzar las metas propuestas.

Esta técnica administrativa se utiliza en muchas instituciones educativas de enseñanza primaria y secundaria a lo largo de nuestro país (como requisito del FODA institucional). Por eso, 
nos ha parecido importante realizar un estudio que permita vislumbrar a la luz de una institución en particular, algunas posibles causas que ocasionan el éxito o el fracaso del trabajo por comités.

Los comités operacionalizan las acciones que se tienen que implementar en un centro educativo, para mitigar o erradicar diversas necesidades con la finalidad principal de mejorar la calidad de la educación. Los comités son unidades de trabajo de las que depende, en buena medida, una efectiva administración curricular, de allí su importancia y la relevancia de esta investigación, la cual busca normalizar una serie de recomendaciones que ayuden a las instituciones de enseñanza media a optimizar el funcionamiento de los comités y, con ello, el de todo el centro educativo.

\section{TEORÍA DE LA ADMINISTRACIÓN CURRICULAR}

\section{Delegación de responsabilidades}

La delegación de responsabilidades administrativas es una de las técnicas más utilizadas para la descentralización de la autoridad en una organización. La descentralización se refiere a la tendencia a distribuir la autoridad para la toma de decisiones en la estructura organizativa, de una empresa o institución dada. Puede darse una centralización total de la autoridad (las decisiones están a cargo de una sola persona y no hay necesidad de administradores subordinados) o una descentralización total (todos tienen autoridad para la toma de decisiones, y no se requieren administradores de niveles más altos).

La delegación de la autoridad es fundamental para la existencia de una empresa o institución. Si no hay una descentralización parcial de la autoridad por medio de la delegación, la toma de decisiones de toda la empresa queda a cargo de una sola persona (lo que no es posible, dado que las organizaciones tienen objetivos grupales, no individuales).

El proceso implica cuatro etapas desde el punto de vista teórico:

1. Determinación de resultados esperados para un puesto particular.

2. Asignación de tareas nuevas al puesto.

3. Delegación de la autoridad para el cumplimiento de esas tareas nuevas (es decir, el subordinado tiene total libertad de administrar sus propios recursos, mientras cumpla sus objetivos).

4. Responsabilización de la persona que ocupa el puesto (cumplimiento de las tareas asignadas).

Paralelo al proceso y a la necesidad de delegar la autoridad en una organización funcional, hay una serie de actitudes personales tanto del administrador como del empleado subordinado que será beneficiario de la delegación:

1. Receptividad: disposición a conceder que otras personas pongan en práctica sus ideas. También debe estar abierto a las ideas de los demás.

2. Disposición a ceder: para una delegación eficaz, el administrador debe estar dispuesto a ceder parte de su poder de decisión a un subordinado. Al respecto, Koontz (1998) menciona: “[...] uno de los errores más graves de los administradores que ascienden en la jerarquía ejecutiva es la manía de seguir tomando decisiones que en sentido estricto pertenecen a puestos que ya no les pertenecen [...]”' (p. 38).

3. Disposición a que los demás cometan errores: aunque es cierto que un administrador no debe permitir los errores, es necesario considerar el aspecto humano, el hecho de que todos se 
pueden equivocar y, más bien, la delegación de autoridad es una inversión en su desarrollo personal como empleado. Elementos como la asesoría, el planeamiento adecuado y los objetivos orientan al subordinado en su toma de decisiones para cometer menos errores.

4. Confianza en los subordinados: se debe confiar. Al delegar autoridad, al mismo tiempo, está estableciendo una relación de confianza entre el administrador y el subordinado; y, así, como el subordinado confía en el jefe, el jefe debe confiar en el subordinado si le delega autoridades adicionales que el jefe antes poseía como propias. Un entorno típico es mencionado también por Koontz (1998, p. 41): "[...] los jefes pretenden desconfiar de sus subordinados cuando en realidad se rehúsan a ceder, temen que el éxito de esos pueda obrar en demérito suyo [...]”.

El grado de descentralización de la autoridad tampoco es arbitrario en una organización. Existe una serie de factores (incluido el temperamento y la personalidad del administrador) de organización y de política que pueden cambiar el curso de una decisión de delegación de autoridad en una organización; entre ellos:

1. Costo de la decisión.

2. Deseo de uniformar políticas.

3. Dimensiones y carácter de la organización.

4. Filosofía administrativa.

5. Deseo de independencia.

6. Disponibilidad de administradores.

7. Técnicas de control.

8. Desempeño descentralizado.

\section{El comité como recurso de administración}

El comité es un conjunto de personas encargado, como grupo, de un asunto. La característica de acción grupal es lo que diferencia al comité de otras estructuras organizativas más individualistas.

El comité, como se mencionó en su definición, es un proceso grupal en el cual sus integrantes tienen funciones muy específicas, como si el mismo comité fuera una organización separada y, completamente, autosuficiente. Es fundamental que en los comités, como en cualquier otro equipo de trabajo, haya armonía y relaciones de confianza entre sus miembros, y que estén dispuestos a colaborar por lograr un objetivo común.

En los comités, algunos miembros son seguidores, otros son líderes. Muchos se dedican a la búsqueda y al análisis de la información que fundamentará las decisiones del comité. Hay casos en que hay conflictos dentro del comité y es necesario llegar a un acuerdo (por lo general político) para proseguir con las tareas asignadas (que pueden ser administrativas, de análisis, de toma de decisiones o, simplemente, para deliberar sobre un tema controversial en la organización).

También se habla de la formalidad estructural de los comités: si son parte de la estructura organizacional y tienen autoridad y deberes específicos, se consideran formales, tal y como ocurre con los comités de una institución educativa. Los informales son simples agrupaciones con un objetivo en común.

En el área de la administración educativa, los comités se utilizan ampliamente y, a veces, se abusa de ello para mantener la libertad académica y el recelo administrativo entre profesores y estudiantes, como lo ejemplifica Koontz (1998, p. 43): “[...] en una gran universidad estadounidense, 
más de 300 comités permanentes tienen injerencia en cuestiones administrativas o prestan asesoría en relación con las políticas de la institución [...]".

Es evidente que, aunque algunas empresas cuentan con demasiados comités que bloquean, de alguna manera u otra, la función administrativa eficaz, hay razones bien fundamentadas que justifican su existencia y buen uso, a saber:

1. Deliberación y criterios grupales.

2. Temor al exceso de autoridad personal.

3. Representación de intereses.

4. Coordinación de departamentos y políticas empresariales.

5. Compartimiento de información.

6. Consolidación de la autoridad.

7. Motivación mediante la participación.

8. Evasión de acciones y responsabilidades directas.

Así como hay razones para su buen uso, hay un conjunto de desventajas en la utilización de la estrategia administrativa por comités, que, por lo general, se reflejan en las organizaciones en las que hay comités para todo y bloquean la función administrativa; algunas de ellas son:

1. Alto costo.

2. Negociación del "mínimo común denominador".

3. Tendencia a la autodestructividad.

4. Dispersión de la responsabilidad.

5. Tiranía de algunos.

Con respecto al mal uso de los comités en una organización, es importante tener claro que existen circunstancias en las cuales no se recomienda la práctica de comités. A continuación, se detallan cinco modalidades en las que no hay necesidad o no se deben utilizar:

1. En reemplazo del administrador.

2. Para estudio o investigación.

3. Para decisiones sin importancia en el ámbito organizacional.

4. Para decisiones ajenas a la autoridad de los participantes.

5. Para consolidar una autoridad fragmentada ${ }^{3}$.

\section{El liderazgo en los comités}

Liderazgo es considerado como el arte o proceso de influir en las demás personas, para que se esfuercen voluntaria y entusiastamente en el cumplimiento de metas grupales. Las metas grupales son los objetivos de los comités en el campo administrativo de una organización. El líder tiene cuatro características o ingredientes, como los llama Koontz (1998):

\footnotetext{
La autoridad fragmentada es un fenómeno que se da en las organizaciones en las cuales una decisión no está al alcance total de un administrador. Otro administrador (por lo general, al mismo nivel jerárquico en la organización) sí tiene la autoridad para tomar el resto de la decisión. La solución es una reunión rápida entre ambos administradores para ponerse de acuerdo, o que la decisión sea tomada por el superior jerárquico de ambos, que sí debe tener autoridad para tomar la decisión.
} 
1. Capacidad para hacer uso eficaz y responsable del poder.

2. Capacidad para comprender que los seres humanos tienen diferentes motivaciones en diferentes momentos y situaciones.

3. Capacidad para inspirar a los demás.

4. Capacidad para actuar a favor del desarrollo de una atmósfera conducente a la respuesta, ante las motivaciones y el surgimiento de éstas.

\section{Teorías del liderazgo}

Inicialmente, los líderes eran estudiados según el uso de la autoridad que ejercían sobre su grupo. Desde esta perspectiva, existen líderes autocráticos, democráticos y liberales. El líder autocrático se caracteriza por imponer y esperar el cumplimiento a expensas de otorgar premios y castigos. El líder democrático es aquel que consulta, alienta la participación y permite que los miembros del grupo apoyen sus decisiones o no. El líder liberal hace uso reducido de su poder, ya que concede a los subordinados un alto grado de independencia y autonomía operacional; su labor es, prácticamente, de contacto con el mundo exterior.

En la actualidad, se utiliza la rejilla administrativa para el análisis de las características de los líderes. La rejilla administrativa es un cuadro de 9x9 celdas. El eje vertical se refiere a la preocupación del líder por las personas y el eje horizontal se refiere a la preocupación del líder por el proceso normal de producción. El problema de la rejilla administrativa es que permite identificar, con facilidad, qué tipo de liderazgo ejerce un líder particular, y su efecto sobre el recurso humano y sobre la producción; pero no dice por qué un administrador o un líder se ubica en una u otra parte de la rejilla.

\section{Enfoques situacionales}

Ya se sabe que el líder se ve muy influido por la situación de donde emerge y donde opera. Hay líderes que son eficaces en un área y pésimos en otra, pero no por ello perderán su condición de líderes. Uno de los elementos fundamentales para el liderazgo lo menciona Koontz (1998) en el capítulo 15 de su libro: "[...] los individuos tienden a seguir a aquellos en quienes perciban (ya sea acertado o desacertado) el ofrecimiento de medios para el cumplimiento de sus deseos personales [...]" (p. 445). El líder es aquella persona que analiza los deseos de los integrantes del grupo y hace lo necesario para satisfacerlos. Esto enfatiza el hecho de que un líder siempre será situacional.

\section{Trabajo en equipo}

Un equipo es un conjunto de personas que se necesitan mutuamente para alcanzar objetivos comunes. Todos los equipos son grupos, pero no todos los grupos son equipos, pues la noción de equipo implica el aprovechamiento del esfuerzo colectivo, producido por cada persona en su interacción con las demás. El trabajo en equipo implica un grupo de personas trabajando, de manera coordinada, en la ejecución de un proyecto.

El trabajo de equipos no es solamente la suma de aportes individuales, ya que un grupo de personas trabajando juntas por una misma meta, pero sin ninguna coordinación entre ellos, no forma 
un equipo. En un equipo todos deben responder por el resultado final y no cada uno de sus miembros de forma independiente. Cada miembro del equipo es responsable de un cometido y sólo si todos ellos cumplen su función será posible alcanzar el objetivo final.

Trabajar en equipo implica tener una visión compartida en la que el o los objetivos y funciones estén claros para cada miembro; de lo contrario, los resultados serían un producto individual o de unos pocos participantes.

Los equipos de trabajo más eficientes son aquellos en los que existe una gran cohesión entre sus miembros; existe un sentimiento de equipo, sus miembros se sienten orgullosos de pertenecer a él. La cohesión no es algo que tiende a surgir de forma espontánea, todo lo contrario, el ser humano suele ser individualista, su carácter es básicamente competitivo. Esto implica que es necesario construir la cohesión, y debe ser construida, fundamentalmente, por el líder del grupo.

El éxito de un equipo no es el resultado de la buena suerte o la casualidad, sino de una serie de factores que lo hacen posible, entre ellos:

1. Participación de todos: un equipo es exitoso cuando hay una participación equilibrada de todos los miembros, con la coordinación de un líder.

2. Metas y reglas establecidas: los equipos efectivos definen normas de actuación, procedimientos y políticas desde el principio, ya que una buena estructura permite cumplir mejor con las tareas.

3. Roles y responsabilidades: cada integrante debe ser consciente de sus habilidades, comprender lo que se espera de él, conocer su rol y el de los demás. El líder del equipo debe supervisar que cada uno sepa y cumpla su puesto.

4. Toma de decisiones objetivas: hay que apoyar las decisiones con datos objetivos, de manera tal que se dé un proceso científico.

5. Plan de actuación: ayuda a identificar los pasos clave para alcanzar, con éxito, los objetivos propuestos por los miembros.

6. Autoevaluación e identificación del avance: las reuniones de autoevaluación deben estar dentro de la planificación de actividades grupales. Se debe equilibrar lo positivo y lo negativo, sin ignorar ninguno de los dos aspectos.

7. Complementariedad: cada miembro domina una parcela determinada del proyecto, el enlace de todos estos conocimientos es necesario para sacar el trabajo adelante.

8. Coordinación: el grupo con un líder a la cabeza, debe actuar de forma organizada con el fin de lograr los objetivos.

9. Confianza: cada persona confía en lo que hace el resto de sus compañeros.

10. Comunicación: el trabajo de equipo exige una comunicación abierta entre todos los miembros.

11. Clima favorable: para que el resultado del equipo sea mayor se debe contar con un clima de trabajo cordial y transparente, para que los miembros trabajen en armonía e involucrados con los resultados del conjunto.

12. Resolución de conflictos: los equipos bien manejados tratan el conflicto de forma abierta y democráticamente, y evitan resentimientos que afecten la persistencia y el éxito del equipo.

13. Compromiso: cada miembro se compromete a aportar lo mejor de sí mismo.

14. Funciones y objetivos claros: estos aspectos le dan la razón de ser al equipo, deben ser realistas y alcanzables y conocidos por todos, a partir de la mediación del líder.

El desempeño de un equipo de trabajo puede mejorar notablemente cuando al frente de él se encuentra un auténtico líder, una persona que reúna características como el ser: justo, trabajador, 
exigente pero comprensivo, respetuoso, defensor de su equipo, preocupado por el bienestar del equipo, organizador nato, dedicado, una persona que otorga confianza, un orientador y guía, un innovador y un motivador. Esta última característica es fundamental, las personas trabajan mejor cuando están motivadas, el estímulo y las motivaciones son los dos motores del trabajo en equipo, para hacer a las personas rendir al máximo.

\section{ANTECEDENTES DEL ESTUDIO DE CASO}

Con la finalidad de evaluar la funcionalidad de la estrategia basada en comités en las instituciones de enseñanza media, este trabajo se fundamentó en el estudio de un caso, que por razones de una mayor facilidad de acceso, se circunscribió en la institución educativa (UPJRAR), ubicada en la provincia de San José, cantón de Tibás.

La UPJRAR tiene como misión ocuparse del desarrollo integral de los estudiantes de la comunidad de la Florida de Tibás, procura proporcionarles las oportunidades necesarias para desarrollarse en los campos intelectual, emocional y físico, por medio de personal altamente capacitado en sus campos respectivos.

En cuanto a su visión, la UPJRAR aspira a ser una institución líder en innovaciones educativas, con el objetivo de lograr un mejoramiento sustancial en el rendimiento académico de sus estudiantes. Esta institución deberá preocuparse por las necesidades particulares de sus estudiantes y darles la atención necesaria, para que éstos sean exitosos durante todo el proceso educativo. La proyección comunal, mediante actividades integradoras, será la manera para lograr un interés mayor de los miembros de la comunidad hacia los requerimientos de la institución.

En la UPJRAR se formaron, durante el período lectivo 2006 (de acuerdo con el Plan Anual Institucional), diez comités, cuyo listado y objetivos se presentan a continuación:

1. Comité de mejoramiento ambiental: tiene como objetivo general desarrollar actividades creadoras de conciencia en el buen uso de los recursos biodegradables en el medio ambiente. Como objetivos específicos se señalan los siguientes:

a. Dar seguimiento al programa educativo de reciclaje.

b. Colaborar con el proyecto de aula verde.

c. Confeccionar un mural para la conmemoración del Día mundial del medio ambiente.

d. Controlar la producción de basura en la institución.

2. Comité de bienestar estudiantil: tiene como objetivo general proveer a los niños y las niñas de la institución con mayor necesidad económica y social, de recursos necesarios que les mitigue su problema mientras asisten al centro educativo. Como objetivos específicos se tienen los siguientes:

a. Brindar alimentación variada a estudiantes con necesidades económicas significativas.

b. Recolectar uniformes y útiles escolares para repartir entre estudiantes con necesidades económicas.

c. Coordinar con las diferentes empresas de la comunidad, con el fin de conseguir financiamiento para el comité.

d. Solicitar una cuota voluntaria a la comunidad estudiantil y al personal docente. 
3. Comité cultural y artístico: el objetivo general es organizar y motivar para actividades y concursos que promuevan el desarrollo cultural-artístico de la comunidad estudiantil. Se tienen como objetivos específicos los que se detallan a continuación:

a. Organizar actividades artísticas y culturales dentro de algunas efemérides relevantes.

b. Motivar la participación de los estudiantes, docentes y padres de familia en concursos culturales y artísticos.

c. Fomentar el aprecio por las raíces culturales.

d. Proyectar los grupos artísticos a la comunidad.

4. Comité de actividades sociales: tiene como objetivo general fomentar las relaciones interpersonales de la institución. Como objetivos específicos se señalan:

a. Programar diferentes eventos sociales del personal docente durante el transcurso del año lectivo.

b. Anunciar, mensualmente, los cumpleaños.

c. Celebrar los cumpleaños del personal con una actividad trimestral.

d. Fomentar valores de relación interpersonal.

5. Comité de apoyo educativo: tiene como objetivo general propiciar la puesta en práctica de las adecuaciones curriculares a todos los estudiantes con necesidades educativas especiales. Sus objetivos específicos son los siguientes:

a. Detectar los casos de estudiantes con necesidades educativas especiales que requieren la aplicación de adecuaciones curriculares.

b. Comunicar al personal docente los casos de estudiantes que requieren adecuaciones curriculares.

c. Dar seguimiento a los estudiantes que requieren la aplicación de adecuaciones curriculares.

d. Asesorar al personal docente sobre el tema de las necesidades educativas especiales y las adecuaciones curriculares, así como sobre sus implicaciones legales.

e. Asesorar a padres de familia de estudiantes con adecuación curricular, sobre el tema y sobre los asuntos legales.

6. Comité de deportes: tiene como objetivo general promover la práctica deportiva, los valores de la salud y el ejercicio, la sana competencia, así como de otros de valores como el respeto, la tolerancia, el esfuerzo y el trabajo en equipo. Sus objetivos específicos son:

a. Fomentar la participación deportiva en diferentes disciplinas en el ámbito institucional.

b. Utilizar la práctica deportiva como un medio para el mejoramiento de la calidad de vida.

c. Proyectar y dar a conocer la institución en el nivel regional, mediante juegos estudiantiles.

7. Comité de evaluación: su objetivo general es, ante la realidad académica que enfrenta el sistema educativo, en la cual la deserción escolar y el fracaso escolar alcanzan cifras alarmantes, centrar 
sus acciones en fortalecer estos aspectos que, en última instancia, coartan la realización de metas en la población estudiantil. En este sentido, aparte de ser vigilantes del cumplimiento del Reglamento de Evaluación de los procesos de aprendizaje, sus funciones estarán encaminadas a dotar a los y las docentes de aquellas herramientas que permitan fortalecer nuestra institución educativa, y, más aún, que puedan propiciar aprendizajes significativos a los y las estudiantes. Como objetivos específicos se señalan los siguientes:

a. Capacitar al personal docente sobre aquellas temáticas relacionadas con la medición y la evaluación.

b. Ofrecer información y asesoría al personal docente sobre los lineamientos de la evaluación institucional.

c. Asesorar a los docentes en el tema de las necesidades educativas especiales, las adecuaciones curriculares y las formas de evaluar a los estudiantes.

d. Propiciar el adecuado proceso de evaluación de la conducta de los estudiantes.

e. Vigilar la correcta elaboración de pruebas sumativas y su ejecución.

f. Resolver objeciones que en materia de evaluación formulen los estudiantes, padres de familia, encargados o docentes.

g. Promover la aplicación de planes remediales durante el curso lectivo.

8. Comité de quinto año: su objetivo general es planificar, organizar y ejecutar todo lo referente a la graduación y a las actividades de quinto año. Como objetivos específicos se proponen:

a. Organizar una comisión de padres de familia que sirva de apoyo.

b. Determinar en conjunto la comisión de cuotas y dineros por recaudar.

c. Involucrar a los estudiantes en la planificación de actividades.

d. Cotizar en diferentes lugares para seleccionar las actividades por realizar.

9. Comité de mejoramiento académico en matemática: su objetivo general es mejorar el nivel académico en el área de la matemática. Sus objetivos específicos son:

a. Propiciar una mejor transición de primaria a secundaria en el área de la matemática.

b. Identificar los elementos curriculares de mayor importancia para lograr una mejor transición de primaria a secundaria, en el área de la matemática.

c. Reforzar el área de la matemática para la solución de las pruebas nacionales de sexto, noveno y undécimo año.

10. Comité de la banda: su objetivo general es suplir las necesidades de la banda de la institución. Sus objetivos específicos son:

a. Coadyuvar en la consecución de los materiales musicales necesarios.

b. Coordinar en la organización de las diferentes actividades.

Para analizar la funcionalidad de cada uno de los comités, se utilizó la encuesta como principal técnica de recolección de información, aplicada a una muestra de 20 profesores (dos miembros por cada comité, elegidos de forma seudo aleatoria), a la asistente administrativa y a la directora de la institución. Estos participantes constituyen las fuentes primarias de información. La encuesta 
fue estructurada con preguntas tanto cerradas como abiertas. La fuente secundaria de información utilizada para realizar el estudio fue el Plan Anual Institucional.

Los elementos incluidos en los instrumentos, abarcan aspectos tales como: la percepción existente en el personal docente y administrativo acerca del nivel de colaboración de los miembros de la institución y la efectividad del trabajo en equipo, el nivel de conocimiento de algunos docentes acerca de los objetivos y las funciones que desempeña el(los) comité(s) del(os) cual(es) forma parte, los mecanismos de trabajo y de control, los alcances reales de cada comité, y las recomendaciones que dentro de este contexto algunos docentes y parte del personal administrativo, brindan para mejorar el desempeño de cada comité. Una de las preguntas está orientada a obtener información cuantificable sobre características fundamentales del trabajo en equipo, tales como la distribución de actividades y el tiempo que le dedica cada persona a cada uno de los comités de los cuales forma parte.

Los resultados arrojados en las encuestas se organizaron mediante porcentajes contando el número de ítemes marcados por cada participante y clasificando las respuestas a las preguntas abiertas en grupos de acuerdo con el tipo de respuesta, lo cual nos permitió realizar una comparación con respecto a los planteamientos en el papel de cada comité y la realidad operativa.

El proceso de evaluación desarrollado en este trabajo emite juicios de valor en cuanto a las posibles causas de éxito o fracaso de la estrategia administrativa basada en comités, en función de las mejores prácticas sustentadas en las experiencias y el fundamento teórico de las autoridades competentes y los resultados del análisis estadístico descriptivo inferido en las encuestas.

\section{ANÁLISIS Y RESULTADOS DEL ESTUDIO DE CASO}

Los resultados de los instrumentos se organizaron por tipo de encuesta, una dirigida a veinte docentes de la UPJRAR y la otra a dos miembros del personal administrativo (la directora y su asistente administrativa). Los datos muestrales se tabularon según la cantidad de respuestas marcadas por ítem y las respuestas abiertas más frecuentes en las preguntas con esta naturaleza.

En la UPJRAR, el 75\% (15 profesores) de los participantes encuestados, consideró que en la institución no existe un clima organizacional que favorezca el trabajo en equipo. Algunas de las causas indicadas por los docentes son las siguientes:

1. Falta de apoyo y control por parte de la dirección. En una organización es fundamental el control y la orientación por parte de las altas esferas del organigrama, y la dirección de una institución educativa debe cumplir con esta labor.

2. Mala comunicación entre el personal docente y el personal administrativo, lo que se destaca con el ausentismo de respuestas por parte de la dirección al planteamiento de propuestas de los docentes. Lo anterior refleja una deficiencia en la comunicación descendente de la UPJRAR.

3. Falta de liderazgo para propiciar el trabajo en equipo, el estímulo y el reconocimiento de logros. El liderazgo permite el logro de objetivos grupales mediante un conjunto de personas individuales, ya que logra "amarrar" los deseos de todos hacia un único fin.

Por el contrario, el personal administrativo encuestado considera que el clima organizacional sí favorece el trabajo en equipo, lo cual denota una descontextualización con la realidad institucional, pues los docentes en su mayoría, opinan lo contrario.

Al cuestionar a los participantes en cuanto a la colaboración tanto de docentes como de administrativos para cumplir, satisfactoriamente, las funciones y el logro de los objetivos de cada 
comité, el 85\% (17 profesores) de los docentes opinó que la colaboración por parte de los colegas y del personal administrativo es insuficiente, e indican las siguientes causas:

1. No hay un verdadero compromiso, interés y motivación por parte de los integrantes de los comités. Este factor tiene una relación directa con lo manifestado por los docentes en cuanto al clima organizacional, lo que reafirma la percepción que tienen acerca de una ausencia de estímulos y de motivación.

2. No existen lineamientos claros por parte de la dirección sobre la organización y el funcionamiento de los comités dentro de la institución. Desde este punto de vista, los docentes tienen la percepción de una falta de orientación y de guía; muchos consideraron en la encuesta una nula supervisión y apoyo de la dirección hacia los comités.

3. No se realiza en los comités una adecuada división de las labores con base en las capacidades de cada integrante. Es un hecho que cada uno de los integrantes de los comités es diferente y, por tanto, puede o debe tener funciones distintas, de acuerdo con un reparto de actividades de manera equitativa. Es injusto cuando en algunos comités hay integrantes que no hacen absolutamente nada y reciben todo el crédito, sólo por haber trabajado como parte del equipo.

4. No hay un real conocimiento acerca del entorno institucional en el que actúa cada comité. Esto incide en los objetivos, en la misión y en la visión de los comités, lo que puede llegar a desvirtuar su función.

Por otra parte, el personal administrativo manifestó en un 100\% una percepción positiva en relación con el trabajo por comités. Algunas de las razones señaladas por la directora y su asistente administrativa fueron las siguientes:

1. Consolidan el quehacer educativo como un proceso integral (si se trabaja de acuerdo con las expectativas de los comités).

2. Logran los objetivos institucionales.

Destacaron, además, la problemática existente en términos operacionales de algunos comités, los que, usualmente, al finalizar el año, no logran sus objetivos, lo que contribuye al deterioro, estancamiento o retroceso de las labores que se realizan dentro de la institución.

Nótese que hay diferencias muy contrastantes entre la opinión de los docentes y la opinión de los administrativos. Quizás las diferencias se deban a que los administrativos lo visualizan según la intencionalidad de su gestión; sin embargo, estos resultados representan una fuerte llamada de atención a la dirección, la cual, al estar inmersa en una estructura organizacional jerarquizada, tiene la obligación de tomar las decisiones y las medidas correspondientes para propiciar un trabajo de equipo exitoso.

Con respecto al trabajo en equipo, el $40 \%$ (8 profesores) de los docentes encuestados, consideró que es inexistente. Como algunas posibles causas señalaron las siguientes:

1. Falta de control y de autoridad por parte de la dirección de la institución, lo cual ocasiona que cada comité trabaje como desee, sin que haya fiscalización del cumplimiento de los objetivos propuestos y de la magnitud de los logros alcanzados. Respecto a esto, el personal administrativo manifestó todo lo contrario, consideran que sí existen mecanismos de control, entre los cuales señalan: 
a. Exposición por escrito y verbal ante el Consejo de Docentes de las metas alcanzadas o que se dejaron sin cumplir.

b. Realización de actividades que han implicado la participación directa de los comités, como responsables de eventos específicos. Entre ellos: la preparación de la Semana Cívica, la Campaña de reciclaje y la Semana de deportes.

2. Presencia de una deficiente comunicación ascendente, descendente y lateral. Muchos docentes consideraron que la comunicación con el personal administrativo, ya sea de forma verbal o escrita, es inadecuada, principalmente, por no recibir respuestas a sus planteamientos; por esta razón, algunos manifestaron que han optado por tomar ellos mismos las decisiones, sin realizar ningún tipo de consulta a la dirección. Asimismo, gran cantidad de profesores expresaron en la encuesta que algunos de los miembros de los comités responsables de comunicar los avances a la dirección, lo hacen de una forma personalizada, con el objetivo de defender sus intereses, sin pensar en el esfuerzo y el beneficio de todo el equipo.

3. Presencia del individualismo en algunos miembros. El individualismo es nocivo en el desarrollo exitoso de un trabajo en equipo, ya que va en detrimento de los objetivos comunes.

4. Retención del poder (autoridad) por parte de algunos de los miembros de los comités, en búsqueda de beneficios personales.

Al analizar los resultados obtenidos por medio de la pregunta número cuatro de la encuesta realizada a los docentes (si se consideraba que todos los docentes trabajaban por igual), es posible concluir que no se da un trabajo de equipo adecuado en los comités de la UPJRAR, por los siguientes factores:

1. No hay una distribución adecuada de la cantidad de trabajo para cada miembro del comité. No se llevan a cabo suficientes reuniones periódicas para distribuir las labores por realizar, razón por la cual se recarga el trabajo de los comités en unos pocos miembros.

2. No se realizan reuniones periódicas para la valoración de los avances (precisamente, porque no hay trabajo que valorar). No hay una eficiente distribución de las actividades. Se revela la presencia del individualismo y la ausencia de un líder interno, que coordine y defina roles y responsabilidades.

3. No se ejerce un control institucional para garantizar el desempeño efectivo de los comités. La dirección no muestra interés en la realización de reuniones periódicas para la coordinación de las actividades, y la comunicación de los logros obtenidos y los resultados producidos.

De forma paradójica, el 100\% del personal administrativo encuestado, consideró bueno el desempeño general de los comités, a pesar de haber manifestado la ausencia de reuniones periódicas con la dirección para coordinar las actividades y comunicar los resultados.

En relación con la definición de objetivos realistas y alcanzables, los docentes manifestaron una percepción positiva, aunque de acuerdo con los resultados obtenidos mediante la pregunta número seis de la encuesta (donde cuestiona si todos llevan a cabo su labor), se obtiene una percepción aproximada de un $50 \%$ de docentes que se inclina hacia un bajo cumplimiento de los objetivos propuestos y una media muestral de un $20 \%$ de cumplimiento de objetivos, al finalizar el período lectivo 2006. Al respecto, los docentes indicaron: 
1. Falta de apoyo y de compromiso, por parte de los mismos miembros de los comités.

2. Ausencia de mecanismos de control. Es importante resaltar el énfasis que los docentes hicieron, constantemente, en la encuesta, ante este factor.

3. Falta de motivación y de estímulos para hacer el trabajo por parte de la dirección.

4. Ausencia de asesoramiento al personal docente para cumplir con los objetivos de cada comité.

5. Falta de recursos físicos y económicos para cumplir con lo estipulado.

Para determinar si los integrantes de cada comité, conocían los objetivos y funciones de su equipo de trabajo o no, se formuló la pregunta cinco de la encuesta realizada a los docentes (labor de los coordinadores en convocar a reuniones periódicas de llevar a cabo la información para la acción de los planes). Mediante una comparación de las respuestas brindadas por los docentes; con los planes de actuación formalizados en el Plan Anual Institucional del año 2006, se llega a la conclusión de que el $50 \%$ de los docentes conocían los objetivos y las funciones de su(s) comité(s), mientras que el otro 50\% no manifiestó ningún conocimiento en ese sentido. Desde el punto de vista administrativo, un equipo de trabajo sin objetivos claros, implica la ausencia de un buen liderazgo y una alta probabilidad de fracaso, pues, a juicio de los expertos, los equipos efectivos definen metas y normas que los dotan de una estructura; para cumplir mejor con sus tareas.

En términos generales, podemos concluir que los docentes de la UPJRAR consideran alcanzables la mayor parte de las metas propuestas de su(s) comité(s) en el Plan de Trabajo Institucional; sin embargo, al mismo tiempo, manifiestan que no serán alcanzadas más de la mitad. A nuestro juicio, la razón de ello puede obedecer a la existencia de un sesgo al contestar los participantes esta pregunta (dado que el $50 \%$ no conocía los objetivos de su equipo de trabajo), o bien, nos muestra un indicador de que el bajo cumplimiento de metas en los comités, está relacionado, principalmente, con una apatía y resistencia en el clima organizacional de la institución. Como investigadores nos abocamos por la segunda opción, al analizar las respuestas de los(as) profesores(as) en las otras preguntas de la encuesta.

De las recomendaciones más importantes indicadas por los docentes para mejorar el desempeño de su(s) comité(s), se destacan las siguientes:

1. Formar comités de tres miembros.

2. Realizar reuniones periódicas, tanto entre miembros del comité como con la dirección, para que, así, exista mayor coordinación, control y participación administrativa.

3. Aumentar la motivación y el estímulo al personal docente involucrado en los comités, para lograr más compromiso en las actividades propias de cada grupo.

4. Mejorar la orientación y la asesoría de la dirección, con respecto a los objetivos y las funciones de cada comité.

5. Analizar y localizar nuevas fuentes de financiamiento.

6. Realizar una adecuada división del trabajo, según las capacidades de cada miembro.

El personal administrativo, por otra parte, sugiere las siguientes recomendaciones:

1. Llevar a cabo reuniones mensuales ante el Consejo de Docentes. Se requiere una explicación pormenorizada de lo que han hecho los comités; cuáles son las metas cumplidas y cuáles los logros por alcanzar.

2. Integrar los comités de acuerdo con las preferencias de los docentes, ya que así trabajarán más a gusto, y se obtendrán (en teoría) mejores resultados. 
3. Dedicar, al finalizar el curso lectivo, durante una semana, un día a cada comité para que sus miembros expongan los logros alcanzados y las metas sin cumplir; además de una proyección del trabajo para el siguiente año.

\section{INDICADORES DE ÉXITO O DE FRACASO EN LA ESTRATEGIA ADMINISTRATIVA BASADA EN COMITÉS}

Como producto de este análisis e interpretación de resultados, se observa que los docentes de la UPJRAR tienen una percepción negativa, en relación con las posibilidades que les brinda el clima organizacional de la institución, para favorecer el trabajo en equipo. Dentro de las causas mencionadas podemos citar: la falta de apoyo y de control de la dirección, una mala comunicación ascendente y descendente, individualismo, retención del poder por parte de algunos docentes y la falta de liderazgo mostrada por el personal administrativo. Esta falta de liderazgo se evidencia en la descontextualización del personal administrativo en relación con el clima organizacional existente en la institución, para promover el trabajo en equipo exitoso.

Los(as) profesores(as) de la UPJRAR tienen una percepción positiva respecto al trabajo en equipo dentro de cada comité; sin embargo, mencionan la presencia de diversos problemas que inciden en la poca colaboración de algunos de sus miembros, tales como: ausencia de un líder que coordine, fiscalice y asigne responsabilidades, falta de motivación por parte de algunos integrantes, falta de lineamientos claros sobre la organización y el funcionamiento de los comités, ausencia de reuniones periódicas para distribuir labores, valorar avances y comunicar resultados a la dirección, ausencia de mecanismos de control por parte del personal administrativo, presencia de una mala comunicación lateral (entre iguales) y falta de conocimiento de algunos miembros acerca de las funciones y objetivos de su(s) comité(s).

Si se toma en consideración la opinión de los expertos y pese a la percepción positiva que tienen los docentes, se concluye, con base en los indicadores anteriores, que en la UPJRAR el trabajo en equipos no es exitoso; dado que: no todos participan, no todos conocen las metas y las normas propuestas, no hay una definición clara de roles y de responsabilidades, no hay coordinación permanente, no hay confianza en el trabajo de los demás, no hay una buena comunicación y no hay un compromiso generalizado.

Es interesante notar cómo los docentes de la UPJRAR consideran alcanzables y realistas los objetivos propuestos por su(s) comité(s) en el Plan Anual Institucional; sin embargo, no perciben que se logre alcanzar más del 50\%, razón por la cual, en la institución, hay un serio problema de resistencia a la autoridad y cierta desmotivación.

Como producto de este análisis e interpretación, hemos logrado también, inferir algunos indicadores que reflejan el mal ejercicio de la estrategia administrativa basada en comités. Los indicadores detectados con el desarrollo de este trabajo se mencionan a continuación:

1. Falta de apoyo entre los compañeros y la dirección.

2. Falta de un liderazgo para la calidad, entendiendo éste como la ciencia de conducir una organización de la situación actual a una situación futura, comparativamente mejor, en la que las personas son el factor principal de cambio, todo esto dentro de un clima organizacional agradable.

3. Mala comunicación ascendente, descendente y lateral. 
4. Ausencia de mecanismos de control, tanto externos (dirección) como internos (coordinador del comité).

5. Presencia del individualismo y recelo por el poder en beneficio personal.

Nuestra labor no ha consistido en determinar si estos indicadores son un mínimo común denominador en un porcentaje significativo de instituciones educativas a lo largo de todo el territorio nacional; pese a ello, nos atreveríamos a conjeturar que algunos de estos aspectos son compartidos por muchas instituciones de enseñanza.

Siendo ésta, a manera de hipótesis, una realidad compartida, es responsabilidad nuestra, también, indagar cómo sería posible mejorar la utilización de la estrategia administrativa basada en comités. Respecto a esto, proponemos una serie de recomendaciones que podrían sugerir una respuesta:

1. Designar comités con, a lo sumo, cuatro miembros y a gusto del personal docente, con la finalidad de facilitar el trabajo de grupo y la participación de todos en las actividades programadas.

2. Designar, en cada comité, un coordinador que, de forma consciente, tome en cuenta la misión y la visión de la organización, los objetivos y las funciones del comité, y reúna ciertas características de liderazgo, entre ellas que: focalice en las personas, inspire confianza, tenga una perspectiva a largo plazo, promueva ideas, promueva cambios, estimule acciones apropiadas, delegue el poder de toma de decisiones y piense en un sistema humano y dinámico.

3. Tomar como punto de partida los intereses y las capacidades de cada miembro, para realizar una adecuada división del trabajo en cada comité.

4. Llevar a cabo reuniones periódicas entre los miembros de cada comité para realizar la distribución equitativa de las labores, discutir los avances y definir los logros.

5. Realizar reuniones periódicas con el personal administrativo, con la finalidad de obtener guía y aprobación de las tareas realizadas, además de informar anomalías en los procesos e irresponsabilidades de aquellos miembros poco comprometidos con su labor.

6. Divulgar, mensualmente, los logros y los avances de cada uno de los comités en el Consejo de Profesores.

7. Entregar un informe verbal y por escrito de cada uno de los comités a la dirección al finalizar el período lectivo, que incluya: los objetivos alcanzados, las metas sin cumplir y su justificación, y una propuesta de trabajo para el siguiente año, con alternativas de solución a los problemas presentados.

8. Buscar estrategias, por parte del personal administrativo, para aumentar la motivación y el estímulo al personal docente involucrado en los comités, con la finalidad de esperar el máximo rendimiento. Algunas de estas estrategias puden ser: económicas, reconocimiento por el alcance de metas parciales, brindar autonomía a los equipos de trabajo, brindar posibilidades de desarrollo profesional, propiciar buenas condiciones laborales, entre otras.

9. Analizar y localizar con ayuda de la dirección, fuentes de financiamiento para realizar las actividades de cada uno de los comités.

La diversidad de contextos dentro del sistema educativo costarricense, demanda un compromiso serio y continuo para lograr objetivos y buscar un mejoramiento en la calidad de los procesos educativos, esto anudado a la crisis financiera y al crecimiento demográfico, exige la búsqueda y el planteamiento de estrategias que optimicen recursos y esfuerzos. 
La estrategia administrativa basada en comités, puede otorgar a las instituciones educativas un medio eficaz para alcanzar objetivos concretos ante las necesidades educativas y organizacionales inmediatas, en períodos de tiempo a un mediano y corto plazo. Lamentablemente, la aplicación de esta estrategia dentro de la realidad educativa costarricense, apunta a un deterioro de su concepción teórica, quedando relegada al cumplimiento de un requisito que se queda en el papel; el FODA institucional. El cambio es necesario, está en nuestras manos crear una conciencia colectiva para asumir la responsabilidad social que todos y todas, como educadores, tenemos en ese sentido.

\section{REFERENCIAS BIBLIOGRÁFICAS}

Koontz, H. \& Weihrich, H. (1998). Administración: una perspectiva global. México: McGrawHill Interamericana.

Murillo, C. (1995). Ensayos sobre desarrollo. San José, Costa Rica: EFUNA.

\section{OTRAS FUENTES CONSULTADAS}

Alba, A. (1994). Currículum: crisis, mito y perspectivas. México: Universidad Nacional Autónoma.

Aguerrondo, I. (1996). La Escuela como una organización inteligente. Buenos Aires, Argentina: Troquel.

Arias, R. (1994). Desafíos de la planificación educativa. Costa Rica: División de Planeamiento y Desarrollo Educativo. San José, Costa Rica: Servicio de documentación del departamento de planes y programas del MEP.

Bolaños, G. \& Molina, Z. (1995). Introducción al currículum. San José, Costa Rica: EUNED.

Costa Rica. Ministerio de Educación Pública (MEP) Consejo Superior de Educación. (1994). Política educativa hacia el siglo XXI. San José, costa Rica: Autor.

Doryan, E. (1995). Una propuesta de un proyecto educativo nacional hacia el año 2005. San José, Costa Rica: Departamento de publicaciones del Ministerio de Educación Pública.

Gurriarán, J. (1994). La planificación educativa en el futuro. Costa Rica: División de Planeamiento y Desarrollo Educativo. San José, Costa Rica: Servicio de documentación del departamento de planes y programas del MEP.

Mayer, F. (1967). Historia del pensamiento pedagógico. Buenos Aires, Argentina: Kapelusz.

Meza, I. \& Pascual, E. Curriculandia. (2004). Material de apoyo curso Diseño Del Currículo. Costa Rica: Maestría en Tecnología e Informática Educativa. 
Richard, D. (2000). Teoría y diseño organizacional. México: International Thomson Editores.

Sarramona, J. (1994). Fundamentos de educación. Barcelona, España: CEAC.

Sarramona, J., Fernández, A. y Tarín, L. (1995). Tecnología didáctica. Barcelona, España: CEAC.

Soto, J. \& Bernardini, A. (1998). La educación actual y sus fuentes filosóficas. (5 ${ }^{\text {a }}$ Reimpres. de la $2^{\mathrm{a}}$ ed.). San José, Costa Rica: EUNED. 